\title{
Quantifying Tripartite Entanglement of Three-Qubit Generalized Werner States
}

\author{
Jens Siewert \\ Departamento de Química Física, Universidad del País Vasco UPV/EHU, 48080 Bilbao, Spain \\ and IKERBASQUE, Basque Foundation for Science, 48011 Bilbao, Spain \\ Christopher Eltschka \\ Institut für Theoretische Physik, Universität Regensburg, D-93040 Regensburg, Germany
}

(Received 13 January 2012; published 4 June 2012)

\begin{abstract}
Multipartite entanglement is a key concept in quantum mechanics for which, despite the experimental progress in entangling three or more quantum devices, there is still no general quantitative theory that exists. In order to characterize the robustness of multipartite entanglement, one often employs generalized Werner states, that is, mixtures of a Greenberger-Horne-Zeilinger (GHZ) state and the completely unpolarized state. While two-qubit Werner states have been instrumental for various important advancements in quantum information, as of now there is no quantitative account for such states of more than two qubits. By using the GHZ symmetry introduced recently, we find exact results for tripartite entanglement in three-qubit generalized Werner states and, moreover, the entire family of full-rank mixed states that share the same symmetries.
\end{abstract}

DOI: 10.1103/PhysRevLett.108.230502

PACS numbers: 03.67.Mn, 03.65.Ud

Currently we observe an impressive evolution in the field of quantum engineering. The state of the art includes up to 14 entangled trapped ions, eight entangled photons, and seven superconducting circuits [1-3]. As opposed to this, entanglement theory is, despite significant progress during the past decade [4,5], still far from adequately quantifying entanglement in multipartite systems, even as small as three qubits.

For few qubits there are well-established entanglement measures [6] that quantitatively characterize class-specific entanglement (that is, classes with respect to stochastic local operations and classical communication, so-called SLOCC classes). There is the concurrence [7] for pure two-qubit states and the three-tangle for three qubits [8]. It is possible to construct analogous measures also for four and more qubits (e.g., Ref. [9]). All of these measures can be extended to mixed states via the convex roof [10]. The difficulty, however, is to compute the convex roof in practice. To date, this is known only for two qubits [11] and a few three-qubit problems chiefly of academic interest $[12,13]$.

Here we provide an exact quantitative description of three-qubit entanglement in mixed states of practical relevance, the generalized Werner state [14,15], and all states with the same symmetries. Precise quantitative knowledge on two-qubit Werner states has triggered important advances, e.g., regarding the relation between entanglement and nonlocality [14], entanglement purification, and noisy quantum channels $[7,16]$, or mixed state entanglement under symmetries $[17,18]$. In contrast, for three-qubit generalized Werner states the exact SLOCC classification has been completed only recently by introducing the concept of Greenberger-Horne-Zeilinger (GHZ) symmetry [19].
Now we apply these results to obtain the exact three-tangle for those states.

We proceed as follows: first we analyze the simple case of GHZ-symmetric two-qubit states for which we have full quantitative knowledge due to the Wootters-Uhlmann method $[11,20]$. We derive the convex roof for the concurrence without referring to that method. Instead we use the approach of the convex characteristic curve [21]. Subsequently we show that this procedure-GHZ symmetrization and quantification via the convex characteristic curve-can be generalized to the three-qubit case and present exact results for the three-tangle, in particular for the three-qubit generalized Werner states.

GHZ symmetry for two qubits.-In the following two sections we will consider exclusively states of two qubits. A common choice for a maximally entangled two-qubit basis is given by $\left|\Phi^{ \pm}\right\rangle=\frac{1}{\sqrt{2}}(|00\rangle \pm|11\rangle)$ and $\left|\Psi^{ \pm}\right\rangle=$ $\frac{1}{\sqrt{2}}(|01\rangle \pm|10\rangle)$. The Werner state

$$
\rho=p\left|\Phi^{+}\right\rangle\left\langle\Phi^{+}\right|+(1-p) \frac{1}{4} \mathbb{1}_{4}
$$

(where $\frac{1}{4} \mathbb{1}_{4}$ is the completely mixed two-qubit state and $0 \leq p \leq 1$ ) has the following symmetries which, as we will see, can be generalized to higher qubit number: invariance under (i) qubit permutations, (ii) simultaneous flips of both qubits (i.e., application of $\sigma_{x} \otimes \sigma_{x}$ ), and (iii) $z$ rotations of the form

$$
U^{2 \mathrm{qb}}(\varphi)=e^{i \varphi \sigma_{z}} \otimes e^{-i \varphi \sigma_{z}}
$$

(where $\sigma_{j}, j=x, y, z$ denote the Pauli matrices). Now let us find the family of all mixed states $\rho^{\mathrm{S}}$ that obey the symmetries (i)-(iii), which we will call henceforth "GHZsymmetric states." The only nonvanishing matrix elements 
are $\rho_{00,00}^{\mathrm{S}}=\rho_{11,11}^{\mathrm{S}}, \quad \rho_{01,01}^{\mathrm{S}}=\rho_{10,10}^{\mathrm{S}}$, and the real offdiagonal elements $\rho_{01,10}^{\mathrm{S}}=\rho_{10,01}^{\mathrm{S}}$. Due to the normalization constraint, the family is characterized by only two real parameters:

$$
x\left(\rho^{\mathrm{S}}\right)=\frac{1}{2}\left[\left\langle\Phi^{+}\left|\rho^{\mathrm{S}}\right| \Phi^{+}\right\rangle-\left\langle\Phi^{-}\left|\rho^{\mathrm{S}}\right| \Phi^{-}\right\rangle\right]
$$

and

$$
y\left(\rho^{\mathrm{S}}\right)=\frac{1}{\sqrt{2}}\left[\left\langle\Phi^{+}\left|\rho^{\mathrm{S}}\right| \Phi^{+}\right\rangle+\left\langle\Phi^{-}\left|\rho^{\mathrm{S}}\right| \Phi^{-}\right\rangle-\frac{1}{2}\right],
$$

whose scale we choose such that the Hilbert-Schmidt metric $d(A, B)^{2} \equiv \frac{1}{2} \operatorname{tr}(A-B)^{\dagger}(A-B)$ coincides with the Euclidean metric in the $x y$ plane. The family of $\rho^{\mathrm{S}}$ is equal to the set of all convex combinations of the three states $\left|\Phi^{+}\right\rangle\left\langle\Phi^{+}|,| \Phi^{-}\right\rangle\left\langle\Phi^{-}\right|$, and $\frac{1}{2}\left(\left|\Psi^{+}\right\rangle\left\langle\Psi^{+}|+| \Psi^{-}\right\rangle \times\right.$ $\left.\left\langle\Psi^{-}\right|\right)$and can be represented by a triangle in the $x y$ plane (Fig. 1).

Concurrence for two-qubit GHZ-symmetric states.Entanglement in pure two-qubit states $|\psi\rangle$ can be quantified by the concurrence $C=\left|\left\langle\psi^{*}\left|\sigma_{y} \otimes \sigma_{y}\right| \psi\right\rangle\right|$ and for mixed states $\rho$ through the convex-roof extension [10]

$$
C(\rho)=\min _{\text {all decomp. }} \sum p_{j} C\left(\psi_{j}\right),
$$

i.e., the minimum average concurrence taken over all possible decompositions $\rho=\sum p_{j}\left|\psi_{j}\right\rangle\left\langle\psi_{j}\right|$ into pure states. There exists always at least one optimal decomposition $\left\{p_{j}^{\text {opt }}, \psi_{j}^{\text {opt }}\right\}$ of $\rho$ for which the average concurrence is equal to the minimum $C(\rho)$.

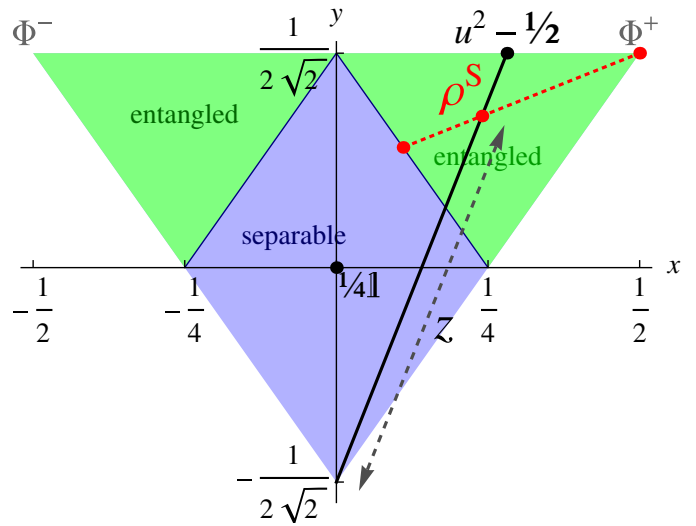

FIG. 1 (color online). The convex set of GHZ-symmetric density matrices $\rho^{S}$ for two qubits. The Bell states $\Phi^{+}$and $\Phi^{-}$form the upper corners of the triangle, while the equal mixture of $\Psi^{+}$and $\Psi^{-}$defines the lower corner. The completely mixed state $\frac{1}{4} \mathbb{1}_{4}$ is located at the origin. The border between separable and entangled states is given by the separability line $y^{\text {sep }}= \pm\left(\frac{1}{2 \sqrt{2}}-\sqrt{2} x\right)$. The solid black line shows an example for the directions characterized by $u$ along which we parametrize the concurrence in Eq. (7). The parameter $z$ gives the position on that line. Further, the red dotted line illustrates the convex combination for the decomposition (9) of the arbitrary state $\rho^{\mathrm{S}}\left(x_{0}, y_{0}\right)$ indicated by a red dot.
In order to calculate the concurrence for two-qubit GHZ-symmetric states $\rho^{\mathrm{S}}$, we apply the method of the convex characteristic curve [21], which relies on the fact that all states $\psi_{j}^{\text {opt }}$ of the optimal decomposition are linear combinations of the elements of any other pure-state decomposition of $\rho$. From such superpositions we determine first a lower bound for the concurrence of $\rho$ that exists by virtue of the restrictions on a pure state $\phi$ from being part of a decomposition of $\rho$. In the second step, we show that this lower bound actually equals the correct concurrence.

The method of convex characteristic curves is easiest applied to a one-parameter manifold. Therefore we consider special directions in the $x y$ plane along which we determine these curves. An appropriate choice are all straight lines starting at the lower corner of the triangle $(x=0, y=-1 /(2 \sqrt{2})$ ) connecting it with the upper horizontal line (Fig. 1). Each straight line is defined by its parameter $u$, whose geometrical meaning is that the $x$ coordinate of the intersection with the horizontal line is given by $x=u^{2}-1 / 2$. A convenient parametrization for the states $\phi$ in the decompositions of GHZ-symmetric twoqubit states $\rho^{\mathrm{S}}$ along the $u$ direction is then

$$
|\phi\rangle=\sqrt{z}\left[u\left|\Phi^{+}\right\rangle+v\left|\Phi^{-}\right\rangle\right]-\sqrt{1-z}[a|01\rangle+b|10\rangle]
$$

with real parameters $u^{2}+v^{2}=1, a^{2}+b^{2}=1$, and $z$ running from 0 to 1 . The corresponding concurrence for a given direction $u$ and fixed value of $z$ is

$$
C(u, z)=\left|z\left(2 u^{2}-1\right)-2 a b(1-z)\right| .
$$

The characteristic curve $\tilde{C}(u, z)$ is obtained by minimizing over $a$. Here, it is not difficult to see that the minimum can be obtained by considering only real parameters $u, v, a$, and $b$ [22]. We find $\tilde{C}(u, z)=\max \left(0,2 z u^{2}-1\right)$ in $u$ direction. The set of curves for all values of $u$ forms a surface that is convex and therefore represents a lower bound to the concurrence [21]. Straightforward coordinate transformation leads to the final two-qubit result (first obtained in Ref. [7])

$$
C(x, y)=\max \left(0,2|x|+\sqrt{2} y-\frac{1}{2}\right) .
$$

This is not just a lower bound-it actually is the concurrence for GHZ-symmetric two-qubit states. We prove this by providing a decomposition that realizes the minimum for each state $\rho^{\mathrm{S}}\left(x_{0}, y_{0}\right)$ above the separability line. An example is

$$
\begin{aligned}
\rho^{\mathrm{S}}\left(x_{0}, y_{0}\right)= & \left(2\left|x_{0}\right|+\sqrt{2} y_{0}-\frac{1}{2}\right)\left|\Phi^{+}\right\rangle\left\langle\Phi^{+}\right| \\
& +\left(\frac{3}{2}-2\left|x_{0}\right|-\sqrt{2} y_{0}\right) \rho^{\mathrm{S}}\left(x_{0}^{\text {sep }}, y_{0}^{\text {sep }}\right),
\end{aligned}
$$

where $\rho^{\mathrm{S}}\left(x_{0}^{\mathrm{sep}}, y_{0}^{\text {sep }}\right)$ is the state at the intersection of the separability line (Fig. 1) and the line that passes through $\Phi^{+}$and $\rho^{\mathrm{S}}\left(x_{0}, y_{0}\right)$. The concurrence of this decomposition 
obviously equals $C\left(x_{0}, y_{0}\right)$. This can be verified by applying the Wootters-Uhlmann method to $\rho^{\mathrm{S}}\left(x_{0}, y_{0}\right)$.

GHZ-symmetry for three qubits.-Now we turn to consider GHZ-symmetric three-qubit states whose properties were described in Ref. [19]. As to the symmetry properties, they are now (ii') invariance under simultaneous flips of all three qubits (i.e., application of $\sigma_{x}^{\otimes 3}$ ) and (iii') invariance under $z$ rotations

$$
U^{3 \mathrm{qb}}\left(\varphi_{1}, \varphi_{2}\right)=e^{i \varphi_{1} \sigma_{z}} \otimes e^{i \varphi_{2} \sigma_{z}} \otimes e^{-i\left(\varphi_{1}+\varphi_{2}\right) \sigma_{z}} .
$$

In full analogy to the two-qubit case, we obtain a family of mixed three-qubit states $\rho^{\mathrm{S}}$ described by two real parameters

$$
x\left(\rho^{\mathrm{S}}\right)=\frac{1}{2}\left[\left\langle\mathrm{GHZ}_{+}\left|\rho^{\mathrm{S}}\right| \mathrm{GHZ}_{+}\right\rangle-\left\langle\mathrm{GHZ}_{-}\left|\rho^{\mathrm{S}}\right| \mathrm{GHZ}_{-}\right\rangle\right]
$$

and

$$
\begin{aligned}
y\left(\rho^{\mathrm{S}}\right)= & \frac{1}{\sqrt{3}}\left[\left\langle\mathrm{GHZ}_{+}\left|\rho^{\mathrm{S}}\right| \mathrm{GHZ}_{+}\right\rangle\right. \\
& \left.+\left\langle\mathrm{GHZ}_{-}\left|\rho^{\mathrm{S}}\right| \mathrm{GHZ}_{-}\right\rangle-\frac{1}{4}\right]
\end{aligned}
$$

with $\left|\mathrm{GHZ}_{ \pm}\right\rangle=(|000\rangle \pm|111\rangle) / \sqrt{2}$. This family can again be represented with a triangle (Fig. 2). The threequbit generalized Werner states are given by

$$
\rho=p\left|\mathrm{GHZ}_{+}\right\rangle\left\langle\mathrm{GHZ}_{+}\right|+(1-p) \frac{1}{8} \mathbb{1}_{8}
$$

with the unpolarized state $\frac{1}{8} \mathbb{1}_{8}$ and $0 \leq p \leq 1$.

The central result of Ref. [19] was the determination of the SLOCC classes of all states within the family, in particular of the boundary $x^{W}(y)$ between $W$ and GHZ class states. The latter (which we refer to as the "GHZ/W line") is given by the parametrized curve

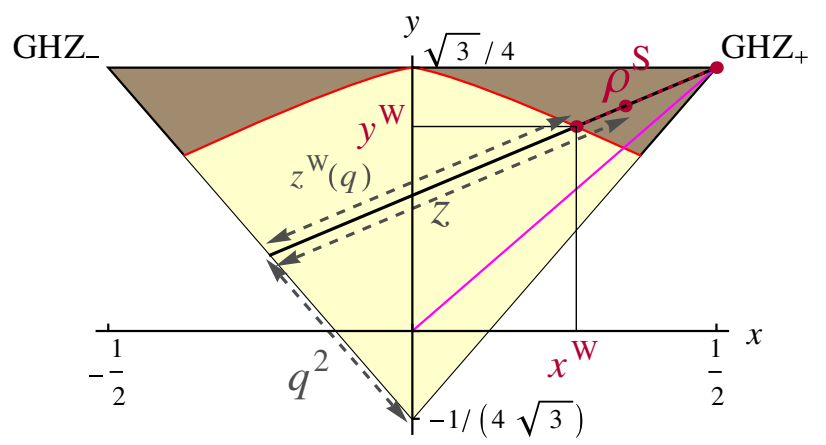

FIG. 2 (color online). The set of GHZ-symmetric three-qubit states [19]. The states $\mathrm{GHZ}_{+}$and $\mathrm{GHZ}_{-}$form the upper corners of the triangle, the lower corner is the symmetrization of $|001\rangle$, and the mixed state $\frac{1}{8} \mathbb{1}_{8}$ is located at the origin. The generalized Werner states lie on the line $y=\frac{\sqrt{3}}{2} x$ (magenta solid line). The border between $W$-class (yellow area) and GHZ-class states (brown area) is given by the $\mathrm{GHZ} / W$ line (red solid line), Eq. (14). The directions along which we parametrize the states using $q$ and $z$ in Eq. (15) are indicated by the black solid line. A state $\rho^{\mathrm{S}}\left(x_{0}, y_{0}\right)$ with nonvanishing three-tangle (19) can be decomposed according to Eq. (18), illustrated by the red dotted line.

$$
x^{W}=\frac{v^{5}+8 v^{3}}{8\left(4-v^{2}\right)}, \quad y=\frac{\sqrt{3}}{4} \frac{4-v^{2}-v^{4}}{4-v^{2}},
$$

where $y \geq \frac{1}{2 \sqrt{3}}$ and $-1 \leq v \leq 1$. We recall that the Werner states are of GHZ type for all $p \geq p_{W}=0.69554 \ldots$

Entanglement of three-qubit GHZ-symmetric states.Our goal is now to quantify the tripartite entanglement in the GHZ-type states of Fig. 2. The appropriate entanglement measure for pure states $\psi$ is the three-tangle [8]

$\tau_{3}=\sqrt{\left|\sum_{j=0, x, z}\left\langle\psi^{*}\left|\sigma_{j} \otimes \sigma_{y} \otimes \sigma_{y}\right| \psi\right\rangle\left\langle\psi^{*}\left|\sigma_{j} \otimes \sigma_{y} \otimes \sigma_{y}\right| \psi\right\rangle\right|}$

with $\sigma_{0} \equiv i \mathbb{1}_{2}$. Its convex-roof extension is defined in analogy with Eq. (5). Note that we use the square root of the original residual tangle in Ref. [8] because then it is a homogeneous function of degree 2 in the coefficients of $\psi$, and its convex roof has peculiar scaling properties that facilitate further application of our results (see Ref. [13]).

In order to find the convex roof of $\tau_{3}$, we use again the convex characteristic curves and follow the procedure that we have applied before to two-qubit states. The starting point is to choose a set of straight lines along which we determine the decompositions of $\rho^{\mathrm{S}}$ and parametrize their elements. It turns out advantageous to decompose $\rho^{\mathrm{S}}$ into $\mathrm{GHZ}_{+}$and the states along the lower left border of the triangle (Fig. 2). The pure states $\phi$ in those decompositions can be represented as

$$
\begin{aligned}
|\phi\rangle= & \sqrt{z}\left|\mathrm{GHZ}_{+}\right\rangle-\sqrt{1-z}\left[q\left|\mathrm{GHZ}_{-}\right\rangle+r(a|001\rangle+b|010\rangle\right. \\
& +c|100\rangle+d|011\rangle+e|101\rangle+f|110\rangle)]
\end{aligned}
$$

with real numbers $0 \leq z \leq 1, q^{2}+r^{2}=1$, and $a^{2}+b^{2}+$ $\ldots+f^{2}=1$. The choice of $q$ fixes the direction and $z$ determines the position on that line. Then we find for the three-tangle of such a state $\phi$

$$
\begin{aligned}
\tau_{3}^{2}(q, z)= & \mid\left[z-(1-z)\left(q^{2}+2 r^{2}(a f+b e+c d)\right)\right]^{2} \\
& -16(1-z)^{2} r^{4}(a f b e+a f c d+b e c d) \\
& -8 \sqrt{2} \sqrt{1-z^{3}} r^{3}(a b d[\sqrt{z}+q \sqrt{1-z}] \\
& +\operatorname{cef}[\sqrt{z}-q \sqrt{1-z}]) \mid
\end{aligned}
$$

which we want to minimize for any fixed values of $z$ and $q$. Due to the symmetry $x \leftrightarrow-x$, we may assume $z \geq 1 / 2$. In analogy with the two-qubit case, it is sufficient to consider only real parameters in Eq. (15) since the minimum of the right-hand side in Eq. (16) is attained for real values of $a, \ldots, f, q, r$ (for the proof, see Ref. [22]). Moreover, the structure of Eq. (16) shows that extremal values obey the conditions $a=b=d$ and $c=e=f$.

The surprising consequence of the preceding argument is that the minimum $\tilde{\tau}_{3}(q, z)$, and thus the characteristic curves, can be found in a one-parameter minimization. The result for each fixed value of $q$ is a function of $z$ that vanishes for $z \leq z^{W}(q)$, where $z^{W}(q)$ is the $z$ value for 


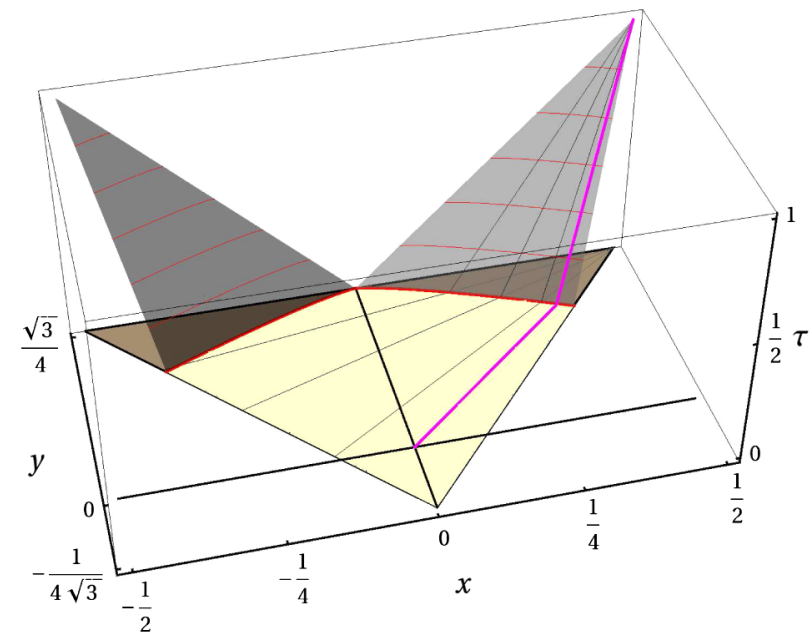

FIG. 3 (color online). The three-tangle for GHZ-symmetric three-qubit states. In the triangle of the states we have used the same colors for the entanglement classes as in Fig. 2. The red solid line in the $x y$ plane represents again the $\mathrm{GHZ} / W$ line, and the magenta solid line the three-tangle of the generalized Werner states. The thin black lines indicate convex characteristic curves $\tau^{*}(q, z)$ for different $q$ values [Eq. (17)].

which the line determined by $q$ intersects the GHZ/W line (Fig. 2). For $z^{W}(q)<z \leq 1$ this function lies above the piecewise linear function

$$
\tau_{3}^{*}(q, z)= \begin{cases}0 & \text { for } 0 \leq z \leq z^{W}(q) \\ \frac{z^{-} z^{W}(q)}{1-z^{W}(q)} & \text { for } z^{W}(q)<z \leq 1 .\end{cases}
$$

Therefore $\tau_{3}^{*}(q, z)$ is the function convex hull of $\tilde{\tau}_{3}(q, z)$ for any fixed value $q$ and represents the convex characteristic curve. The surface formed by the entire set of convex characteristic curves $\tau_{3}^{*}(q, z), 0 \leq q \leq 1$, shown in Fig. 3, is convex from below. Therefore, it fixes at least a lower bound to the three-tangle of the states $\rho^{\mathrm{S}}$.

The final step in our derivation is to show that we can always find a decomposition that realizes the value $\tau_{3}^{*}$ assigned by Eq. (17) to each state $\rho^{\mathrm{S}}$. As before, we provide an example decomposition. Assume we are given an arbitrary state $\rho^{\mathrm{S}}(x, y)$. It has nonvanishing three-tangle only if it is located above the GHZ/W line in Fig. 2. This is because all states in the area below are locally equivalent to the $W$ state or are (bi-)separable and therefore have no three-tangle [19].

Hence assume that $\rho^{\mathrm{S}}(x, y)$ lies above the GHZ/W line. Its $z$ and $q$ values can be computed from the coordinates via $z=x+(\sqrt{3} / 2) y+1 / 8$ and $q=(\sqrt{3} y+$ $1 / 4-z) /(1-z)$. An obvious decomposition is

$$
\begin{aligned}
\rho^{\mathrm{S}}(x, y)= & \frac{z-z^{W}(q)}{1-z^{W}(q)}\left|\mathrm{GHZ}_{+}\right\rangle\left\langle\mathrm{GHZ}_{+}\right| \\
& +\frac{1-z}{1-z^{W}(q)} \rho^{\mathrm{S}}\left(x^{W}(q), y^{W}(q)\right),
\end{aligned}
$$

where $\rho^{\mathrm{S}}\left(x^{W}(q), y^{W}(q)\right)$ is the state at the intersection point of the GHZ/W line with the straight line that contains $\mathrm{GHZ}_{+}$and $\rho^{\mathrm{S}}(x, y)$. The average three-tangle of the decomposition Eq. (18) clearly coincides with the value obtained from the convex characteristic curve $\tau_{3}^{*}(q, z)$.

Consequently, the surface given by $\tau_{3}^{*}(q, z)$ in Eq. (17) is identical to the convex roof of $\tau_{3}$ for GHZ-symmetric three-qubit states and is the main result of our work. We can restate it in terms of the coordinates $(x, y)$ in the following way. Given a GHZ-symmetric three-qubit state $\rho^{\mathrm{S}}$ with coordinates $\left(x_{0}, y_{0}\right)$, one first determines the straight line that connects the $\mathrm{GHZ}_{+}$state at $(1 / 2, \sqrt{3} / 4)$ with the point $\left(x_{0}, y_{0}\right)$. Next, one finds the intersection point $\left(x_{0}^{W}, y_{0}^{W}\right)$ of this line with the GHZ/W line. Then the three-tangle $\tau_{3}\left(\rho^{\mathrm{S}}\left(x_{0}, y_{0}\right)\right)$ is given by

$$
\tau_{3}\left(x_{0}, y_{0}\right)= \begin{cases}0 & \text { for } x_{0}<x_{0}^{W} \text { and } y_{0}<y_{0}^{W} \\ \frac{x_{0}-x_{0}^{W}}{\frac{1}{2}-x_{0}^{W}}=\frac{y_{0}-y_{0}^{W}}{\frac{\sqrt{3}}{4}-y_{0}^{W}} & \text { otherwise. }\end{cases}
$$

The most interesting GHZ-symmetric states are the generalized Werner states. Their three-tangle is

$$
\begin{aligned}
& \tau_{3}^{\text {Werner }}(p) \\
& = \begin{cases}0 & \text { for } p \leq p_{W}=0.69554 \ldots \\
\frac{p-p_{W}}{1-p_{W}} \approx 3.2845 p-2.2845 & \text { for } p_{W}<p \leq 1 .\end{cases}
\end{aligned}
$$

Conclusion.-We have discussed the quantification of mixed-state entanglement in GHZ-symmetric states for two and three qubits by using the method of convex characteristic curves [21]. Our first important result is that we could derive the entanglement in these full-rank mixed states for two qubits without reference to the Wootters-Uhlmann method. This indicates how powerful the approach is. In fact, subsequently we have applied it to three-qubit GHZ-symmetric states and could derive the convex roof of the three-tangle. In particular, we have quantified exactly the tripartite entanglement in three-qubit generalized Werner states, which will help to provide new insight into the quantitative theory of multipartite entanglement.

This work was funded by the German Research Foundation within SPP 1386 (C.E.), and by Basque Government Grant No. IT-472-10 (J. S.). The authors thank J. Fabian and K. Richter for their support.

[1] T. Monz, P. Schindler, J. T. Barreiro, M. Chwalla, D. Nigg, W. A. Coish, M. Harlander, W. Hansel, M. Hennrich, and R. Blatt, Phys. Rev. Lett. 106, 130506 (2011).

[2] X.-C. Yao, T.-X. Wang, P. Xu, H. Lu, G.-S. Pan, X.-H. Bao, C.Z. Peng, C. Y. Lu, Y.A. Chen, and J.W. Pan, Nature Photon. 6, 225 (2012). 
[3] M. Mariantoni, H. Wang, T. Yamamoto, M. Neeley, R. C. Bialczak, Y. Chen, M. Lenander, E. Lucero, A. D. O'Connell, D. Sank, M. Weides, J. Wenner, Y. Yin, J. Zhao, A. N. Korotkov, A. N. Cleland, and J. M. Martinis, Science 334, 61 (2011).

[4] M. B. Plenio and S. Virmani, Quantum Inf. Comput. 7, 1 (2007); R. Horodecki, P. Horodecki, M. Horodecki, and K. Horodecki, Rev. Mod. Phys. 81, 865 (2009).

[5] O. Gühne and G. Tóth, Phys. Rep. 474, 1 (2009).

[6] G. Vidal, J. Mod. Opt. 47, 355 (2000).

[7] C. H. Bennett, D. P. DiVincenzo, J. A. Smolin, and W. K. Wootters, Phys. Rev. A 54, 3824 (1996).

[8] V. Coffman, J. Kundu, and W. K. Wootters, Phys. Rev. A 61, 052306 (2000).

[9] J.-G. Luque and J.-Y. Thibon, Phys. Rev. A 67, 042303 (2003); D. Dokovic and A. Osterloh, J. Math. Phys. (N.Y.) 50, 033509 (2009); S.S. Sharma and N. K. Sharma, Phys. Rev. A 82, 052340 (2010); O. Viehmann, C. Eltschka, and J. Siewert, Phys. Rev. A 83, 052330 (2011).

[10] A. Uhlmann, Open Syst. Inf. Dyn. 5, 209 (1998).

[11] W. K. Wootters, Phys. Rev. Lett. 80, 2245 (1998).

[12] C. Eltschka, A. Osterloh, J. Siewert, and A. Uhlmann, New J. Phys. 10, 043014 (2008); E. Jung, M. R. Hwang, D. K. Park, and J.W. Son, Phys. Rev. A 79, 024306
(2009); S. J. He, X.H. Wang, S. M. Fei, H.X. Sun, and Q. Y. Wen, Commun. Theor. Phys. 55, 251 (2011).

[13] O. Viehmann, C. Eltschka, and J. Siewert, Appl. Phys. B 106, 533 (2012).

[14] R. F. Werner, Phys. Rev. A 40, 4277 (1989).

[15] A. O. Pittenger and M. H. Rubin, Opt. Commun. 179, 447 (2000); W. Dür and J. I. Cirac, Phys. Rev. A 61, 042314 (2000).

[16] C. H. Bennett, G. Brassard, S. Popescu, B. Schumacher, J. A. Smolin, and W. K. Wootters, Phys. Rev. Lett. 76, 722 (1996).

[17] M. Horodecki and P. Horodecki, Phys. Rev. A 59, 4206 (1999).

[18] B. M. Terhal and K. G. H. Vollbrecht, Phys. Rev. Lett. 85, 2625 (2000).

[19] C. Eltschka and J. Siewert, Phys. Rev. Lett. 108, 020502 (2012).

[20] A. Uhlmann, Phys. Rev. A 62, 032307 (2000).

[21] A. Osterloh, J. Siewert, and A. Uhlmann, Phys. Rev. A 77, 032310 (2008).

[22] See Supplemental Material at http://link.aps.org/ supplemental/10.1103/PhysRevLett.108.230502 for details where we prove the statement that in the minimization for the characteristic curves [Eqs. (7) and (16)] it is sufficient to consider only real parameters. 